\title{
Rendimiento Y Componentes De Valor Nutritivo De Tres Cultivares Panicum Máximum
}

\author{
Yield And Nutritive Value Components Of Three Cultivars Panicum Maximum
}

\section{Edgar Alexis Polo L.}

Universidad de Panamá, Panamá. Departamento de Zootecnia, Panamá , Panamá E-mail: epolo61@ hotmail.com. Orcid; 0000-0002-1246-2355

\section{Resumen}

Los géneros Panicum variedades como Mombaça, Tobiatá y Colonial se han introducido a diferentes regiones del país con éxito y fracaso debido a que no se tiene la información de su comportamiento a nivel nacional y nos guiamos por la de los países donde se han introducidos con características de clima, suelo y topografía totalmente variante a la de nuestro país. El trabajo se realizó en el Centro de Enseñanzas e Investigaciones Agropecuarias de Tocumen (CEIAT) de la Facultad de Ciencias Agropecuarias de la Universidad de Panamá. El objetivo de este trabajo fue estudiar tres cultivares de Panicum máximum (Massai, Tanzania y Aruana). La siembra fue con semilla gámica a razón de $6.0 \mathrm{~kg} / \mathrm{ha}$ de semillas gámica, a una distancia de $0.50 \mathrm{~cm}$ entre surco y a chorro continuo. El diseño experimental fue de bloque completamente al azar, con cuatro repeticiones. Los cortes se realizaron cada 30 días. El tamaño de las parcelas fue de $9.00 \mathrm{mt}^{2}$. Aunque las tres especies tuvieron comportamiento aceptable por su rendimiento de materia seca $(P<0.05)$, destaco Panicum maximum cv. Tanzania con 30.00 y 9.40 tha para los periodos lluviosos (invierno) y seco (verano), respectivamente, mientras que Panicum maximum cv. Massai resulto superior $(\mathrm{P}<0.05)$ en el contenido de proteína bruta (11.30\%). En la época seca no se encontraron diferencias significativas en la altura de los cultivares, pero en el periodo lluvioso Panicum maximum cv. Tanzania y Panicum maximum cv. Massai superaron a Panicum maximum cv. Aruana.

Palabras clave: Rendimento, valor nutritivo, Panicum maximum, Massai, Aruana, Tanzania

\section{Abstract}

The genera Panicum varieties like Mombaca, Tobiatá and Colonial have been introduced to different regions of the country with success and failure because they do not have the information of their behavior at the national level and are guided by the countries where they have been introduced with features climate, soil and topography totally variant to our country. The work was performed at the Center for Agricultural Research and Teaching Tocumen (CEIAT), Faculty 
of Agricultural Sciences of the University of Panama. The aim of this work was to study three cultivars of Panicum maximum (Massai, Tanzania and Aruana). Planting was with gamic seed rate of $6.0 \mathrm{~kg} /$ ha of seeds gamic, at a distance of $0.50 \mathrm{~cm}$ between groove and continuous stream. The experimental design was a randomized complete block with four replications, The cut is made every 30 days. The plot size was $9.00 \mathrm{~m} 2$. Although the three species had its dry matter yield $(P<0.05)$ acceptable behavior, emphasized Panicum maximum cv. Tanzania with 30.00 and $9.40 \mathrm{t} / \mathrm{ha}$ for dry rainy seasons (winter) and (summer), respectively, while Panicum maximum cv. Massai was superior ( $P$ $<0.05)$ in crude protein content $(11.30 \%)$. In the dry season there were no significant differences in the height of the cultivars were found, but in the rainy season Panicum maximum cv. Tanzania and Panicum maximum cv. Massai outperformed Panicum maximum cv. Aruana.

Keywords: Yield, nutritive value, Panicum maximum, Massai, Aruana, Tanzania

\section{Introducción}

Los rumiantes en el trópico basan su alimentación en el consumo de forrajes, por ello es necesario determinar tanto el rendimiento como el contenido nutricional, con el fin de estimar productividad y nutrientes que puedan ser aprovechada por el animal.

Sin embargo, su crecimiento y productividad está influida por las condiciones climáticas existentes principalmente por la distribución anual de las lluvias, que unido a otros factores del medio ambiente y de manejo, repercuten en que estos no reflejen totalmente su potencial productivo y nutritivo.

Dichos elementos interactúan y tienen un marcado efecto en el crecimiento de las especies y variedades de pastos en los diferentes meses del año, provocando un desbalance estacional en los rendimientos, que ocasiona un déficit de alimento principalmente en el periodo poco lluvioso. A esta situación hay que añadir que los suelos destinados al cultivo de pastos en su mayoría son de baja fertilidad y mal drenaje, que conjuntamente, con el clima ejercen efectos negativos en la productividad, calidad y persistencia de las especies de pastos (Blanco, 1991).

Los pastos del género Panicum conocidos también como Guinea o Indiana, son de especies perenne de crecimiento erecto, que se desarrolla en plantas aisladas o en matojos. Puede alcanzar hasta tres metros de altura y presenta un buen cubrimiento del terreno cuando se utiliza abundante cantidad de semilla. Las cariópsides poseen una esterilidad alta siendo necesario usar semillas seleccionadas de buena calidad para su propagación. Existe una gran cantidad de variedades desde las muy altas como la Tobiatá de origen brasilero, hasta variedades de tallo delgado como la Trichoglume o variedades enanas del tipo de las llamadas pajaritas y siempre verdes (green leaf) procedentes de Australia (Bernal, J., 1991). 
En los últimos años la introducción de semillas de pastos mejorados ha estimulado a los productores a mejorar sus praderas nativas en busca de mayor productividad de biomasa y a su vez aumentar las cargas animales por hectárea. En el caso de los géneros Panicum variedades como Massai, Tobiatá y Tanzania se han introducido a diferentes regiones del país con éxito y fracaso debido a que no se tiene la información de su comportamiento a nivel nacional y nos guiamos por la de los países donde se han introducidos con características de clima, suelo y topografía totalmente variante a la de nuestro país.

En 2010 se introdujo a Panamá procedente de Brasil a través de la ANAGAN (Asociación Nacional de Ganaderos), el pasto Panicum maximum (Piatá), pasto este que según AGRICAMPO (2011), se adapta a suelos fértiles pudiendo prosperar con buena fertilización, y en suelos no encharcados. Crece en alturas que van desde el nivel del mar hasta los 2,000 m.s.n.m. y en regiones con más de $700 \mathrm{~mm}$. de lluvias. Se caracteriza por ser una pastura para uso de ganado ovino y caprino dado su porte bajo, que se sitúa por debajo de $1 \mathrm{~m}$ de altura, no obstante, es también indicada para bovinos y equinos con excelentes resultados. Produce de 15 a 20 toneladas de materia seca por año, con niveles de proteína en la materia seca de 9 - $12 \%$ con 64 a $66 \%$ de digestibilidad (Agricampo, 2011).

Es por eso el interés de comparar los pastos Panicum máximum de reciente introducción al país para observar sus reales bondades de adaptación producción y persistencia.

\section{Metodología}

El experimento fue realizado en el Centro de Investigación Agropecuaria de Tocumen (CEIAT), ubicado en el sector Este de la provincia de Panamá. Se estudiaron tres cultivares de Panicum máximum (Massai, Tanzania y Aruana). La siembra fue hecha con semilla gámica a razón de $6.0 \mathrm{~kg} / \mathrm{ha}$, a una distancia de $0.50 \mathrm{~cm}$ entre surco y a chorro continuo.

El diseño experimental fue de bloque completamente al azar, con cuatro repeticiones. Las frecuencias de corte fueron a cada 30 días. El tamaño de las parcelas fue de $9.00 \mathrm{mt}^{2}$.

Al momento de la siembra se realizó una fertilización basal con abono completo de la fórmula 12-24-12 a razón de 2.0 qq/ha. A los 120 días de sembrado se realizó un corte de uniformidad a una altura de $25 \mathrm{~cm}$. sobre el suelo y se aplicó fertilización nitrogenada (Urea 46\%), sobre cada parcela al voleo.

En cada uno de los cortes después de desechar los $0.50 \mathrm{~cm}$ de borde se tomaran muestras del material vegetativo por tratamiento, analizando separadamente las muestras y se determinaron la producción de materia seca, porcentajes de proteína cruda, fósforo, potasio, calcio y magnesio. La altura se midió con una regla graduada en centímetros, tomándose desde la base del tallo hasta el punto máximo del área foliar de la planta. Se tomaron muestras de 200 gramos dos veces al año para la composición bromatológica según AOAC (1997). 
Los suelos de Tocumen son de textura franco arcilloso, fertilidad media y fuertemente ácidos. La composición química del suelo del estudio se muestra en el Cuadro 1.

CUADRO 1. COMPOSICIÓN QUÍMICA DEL SUELO DE LAS PARCELAS EXPERIMENTALES. TOCUMEN, PANAMÁ, 2013.

\begin{tabular}{|c|c|c|}
\hline COMPONENTE & VALOR & INTERPRETACIÓN \\
\hline Arena (\%) & 38 & Franco arcilloso \\
\hline Limo (\%) & 24 & \\
\hline Arcilla (\%) & 38 & Ácido \\
\hline Ph & 4.23 & Medio \\
\hline Fósforo (ppm) & 10.8 & Medio \\
\hline Potasio (ppm) & 87 & Alto \\
\hline $\begin{array}{c}\text { Calcio (meq/100 ml de } \\
\text { suelo) }\end{array}$ & 13.1 & Bajo \\
\hline Materia orgánica & 2.87 & . \\
\hline
\end{tabular}

Fuente: Laboratorio de Suelos. FCA. Universidad de Panamá. 2013.

El análisis de los datos se realizó mediante el procedimiento SPSS del paquete Statistical Package for the Social Sciences, y se empleó la comparación múltiple de Duncan. Los parámetros a evaluar fueron: 1- Altura de las plantas (cm), 2- Rendimiento de materia seca (Kg/ha) y 3-Calidad nutritiva de las pasturas (proteína cruda) (\%).

\section{Resultados}

Las especies estudiadas durante la época seca (Tabla 1) tuvieron un buen comportamiento en cuanto al rendimiento de materia seca (MS), siendo superior $(\mathrm{P}<0.05)$ Panicum máximum cv Tanzania sobre Panicum maximum Aruana, y Panicum máximum cv Massai que no muestra diferencias con el cv Aruana. Estos resultados son similares a los alcanzados por los especialistas de EMBRAPA en Brasil (2008). En relación a la altura, no se presentan diferencias entre los cultivares Tanzania y Aruana para este período. Siendo el Massai quien presento el crecimiento menor en el estudio y periodo evaluado.

\section{TABLA 1. COMPORTAMIENTO DE LOS INDICADORES EVALUADOS PARA LOS Panicum máximum EN EL PERIODO SECO 1/}

\begin{tabular}{|c|c|c|}
\hline ESPECIE & MATERIA SECA(t/ha) & ALTURA $(\mathrm{m})$ \\
\hline Panicum máximum cv Tanzania & $9.40 \mathrm{a}$ & $1.98 \mathrm{a}$ \\
\hline Panicum máximum cv Aruana & $6.41 \mathrm{~b}$ & $1.86 \mathrm{a}$ \\
\hline Panicum máximum cv Massai & $8.81 \mathrm{ab}$ & $1.75 \mathrm{~b}$ \\
\hline
\end{tabular}

1/ Valores seguidos de la misma letra no difieren al 5\% de probabilidad según la prueba de medias Duncan.

En la Tabla 2 se muestra el comportamiento de los indicadores evaluados para las especies en el período lluvioso, donde se observa la superioridad en cuanto al rendimiento de materia seca $(\mathrm{P}<0.05)$ del Panicum máximum cv Tanzania 
sobre las otras dos especies, que a su vez no difieren entre sí. Esto nos indica que Panicum máximum cv Tanzania es de las especies de pasto que logra mayor efectividad en esta época, cuando las condiciones de luz, temperatura y humedad son favorables. Con relación a la altura (Tabla 2) los cultivares Tanzania y Aruana superaron significativamente $(\mathrm{P}<0.05)$ a Panicum máximum cv Massai, sin diferir entre ellos. Cabe señalar el gran número de ahijamientos que forma el Massai, mayor que cualquier otro cultivar conocido de Panicum. Los rangos de rendimiento y altura alcanzados por estos Panicum en el periodo Iluvioso son casi similares a los reportados por Rettaly (2009) con los Panicum máximum cv Aries y Atlas en suelos similares al estudiado característico por su baja fertilidad, alta acidez y con concentraciones elevadas de aluminio.

La variabilidad del rendimiento de las especies (de acuerdo a su biología) está relacionada con su adaptación a las condiciones edáficas y climáticas del área de estudio (CIAT, 1992) y su capacidad para ajustar los mecanismos de persistencia a las condiciones más o menos adversas que les impone el medio (Lascano y Euclides, 1998).

\section{TABLA 2. COMPORTAMIENTO DE LOS INDICADORES EVALUADOS PARA LOS Panicum máximum EN EL PERIODO LLUVIOSO 1/}

\begin{tabular}{|c|c|c|}
\hline ESPECIE & MATERIA SECA(t/ha) & ALTURA (m) \\
\hline Panicum máximum cv Tanzania & $30.00 \mathrm{a}$ & $2.11 \mathrm{a}$ \\
\hline Panicum máximum cv Aruana & $20.51 \mathrm{~b}$ & $1.98 \mathrm{a}$ \\
\hline Panicum máximum cv Massai & $22.92 \mathrm{ab}$ & $1.78 \mathrm{~b}$ \\
\hline
\end{tabular}

1/ Valores seguidos de la misma letra no difieren al 5\% de probabilidad según la prueba de medias Duncan.

Con relación a la composición bromatológica (Tabla 3) se encontraron diferencias significativas entre las especies para todos los elementos, destacando el Panicum máximum cv. Massai en sus contenidos de Proteína bruta, calcio y magnesio superando significativamente $(P<0.05)$ a las restantes que no muestran diferencias entre ellas para los dos primeros elementos; sin embargo, el Panicum máximum cv Aruana contiene mayor contenido de magnesio, por lo que aventaja significativamente $(P<0.05)$ al Panicum máximum cv Tanzania.

El contenido de potasio fue superior en el Panicum máximum cv Tanzania y en el Panicum máximum cv Aruana que no mostraron diferencias entre ellos, pero superan $(\mathrm{P}<0.05)$ al Panicum máximum cv Massai.

En cuanto a los niveles de fósforo este fue superior en el Panicum máximum cv Aruana difiriendo significativamente $(\mathrm{P}<0.05)$ de los otros Panicum; no obstante, el bajo contenido de fósforo resulta una limitante desde el punto de vista nutricional y apunta a la necesidad de suplementar a los animales que utilizan estos pastos en su alimentación (Youssef, 1988).

A su vez Cuadrado, Vega y Torregrozza, (1996), indican que niveles de proteína por debajo del $7 \%$ no permite una utilización completa de los carbohidratos del forraje y además, la tasa de pasaje de la digesta disminuye, 
en consecuencia, digestibilidad y consumo voluntario son reducidos significativamente.

Tabla 3. Composición Bromatológica De Las Especies Panicum máximum 1/

\begin{tabular}{|c|c|c|c|c|c|}
\hline ESPECIE & $\begin{array}{c}\text { PROTEINA } \\
\text { BRUTA (\%) }\end{array}$ & $\begin{array}{c}\text { FOSFORO } \\
(\%)\end{array}$ & $\begin{array}{c}\text { POTASIO } \\
(\%)\end{array}$ & $\begin{array}{c}\text { CALCIO } \\
(\%)\end{array}$ & $\begin{array}{c}\text { MAGNESIO } \\
(\%)\end{array}$ \\
\hline $\begin{array}{c}\text { Panicum } \\
\text { máximum } \\
\text { cv Tanzania }\end{array}$ & $7.90 \mathrm{~b}$ & $0.21 \mathrm{~b}$ & $1.50 \mathrm{a}$ & $0.71 \mathrm{~b}$ & $0.17 \mathrm{c}$ \\
\hline $\begin{array}{c}\text { Panicum } \\
\text { máximum } \\
\text { cv Massai }\end{array}$ & $11.30 \mathrm{a}$ & $0.21 \mathrm{~b}$ & $1.08 \mathrm{~b}$ & $0.91 \mathrm{a}$ & $0.25 \mathrm{a}$ \\
\hline $\begin{array}{c}\text { Panicum } \\
\text { máximum } \\
\text { cv Aruana }\end{array}$ & $7.85 \mathrm{~b}$ & $0.23 \mathrm{a}$ & $1.43 \mathrm{a}$ & $0.75 \mathrm{~b}$ & $0.19 \mathrm{~b}$ \\
\hline
\end{tabular}

1/ Valores seguidos de la misma letra no difieren al 5\% de probabilidad según la prueba de medias Duncan.

\section{Conclusión}

Los tres cultivares tuvieron un comportamiento aceptable en las condiciones edafoclimáticas del área donde se evaluaron; se destacó el cultivar Panicum máximum cv Tanzania por sus rendimientos, con 9.40 y 30.00 toneladas de materia seca/hectárea para el período seco y lluvioso, respectivamente y el Panicum máximum cv Massai en contenido proteico con valor de $11.30 \%$.

\section{Recomendaciones}

Realizar más estudios agronómicos en el área de fertilización producción y utilización en la alimentación animal en nuestro país de los cultivares Panicum máximum cv Tanzania y Panicum máximum cv Massai evaluados en esta investigación.

\section{Referencias Bibliográficas}

AGRICAMPO, 2011. Pasto Aruana. Panicum máximum cv. Aruana. Disponible en: http://www.agricampo.com.mx. 29/08/2011.

AOAC. 1997. Official Methods of Analysis (25th Ed.). Washington, DC.; Association of Official Analytical Chemist.

BERNAL, J. 1991. Pastos y Forrajes Tropicales. Producción y Manejo. 2da Edición. Banco Ganadero. Colombia. 543 pag. 
BLANCO, F. 1991. La persistencia y el deterioro de los pastizales. Revista de Pastos y Forrajes. EEPF Indio Hatuey, vol. 14. pag. 87- 103.

CIAT (Centro Internacional de Agricultura Tropical). 1992. Tropical Grasses and Legume: Optimizing Genetic Diversity for Multipurpose Use. Annual Report, Project IP-5. Consultado en 2013, desde htt//www.ciat.ab.cr/bims/ho3/p13/co46/00159/fo1382/g0056248971.h tm.

CUADRADO, H.; VEGA, A. Y TORREGROZZA, L. 1996. Producción, Composición Química y Digestibilidad del pasto Braquipara (Brachiaria arrecta) en diferentes épocas y edad de rebrote. Disponible en: http: // www.corpoica.org.com.

EMBRAPA, 2008. Forrageiras Tropicais. Tecnologia para a Liderança Mundial de Carnes. Empresa Brasileira de Pesquisa Agropecuaria. Gado de Corte. Brasil. Plegables.

LASCANO, C. E. y EUCLIDES, P. B. 1998. Calidad nutricional y Producción Animal en Pasturas. (capítulo 7, pp. 116-135). Cali, Colombia: CIAT.

RETTALY, R. 2009. Utilización de los Pastos Aries y Atlas (Panicum máximum). Pasturas de Elevada Producción de Forraje. Instituto de Investigación Agropecuaria de Panamá. Folleto Técnico. 9 pag.

YOUSSEF, F.G. 1988. Somme Factors Affecting the Mineral Profiles of Tropical Grasses. Outlook of Agriculture, 17 (3), 104-111. 